\title{
Point-particle method to compute diffusion-limited cellular uptake
}

\author{
A. Sozza, ${ }^{1}$ F. Piazza, ${ }^{2}$ M. Cencini, ${ }^{3}$ F. De Lillo, ${ }^{1}$ and G. Boffetta ${ }^{1}$ \\ ${ }^{1}$ Department of Physics, Università di Torino \& INFN, via P. Giuria 1, 10125 Torino, Italy \\ ${ }^{2}$ Centre de Biophysique Moléculaire, CNRS-UPR 4301 and Université d'Orléans, F-45071 Orléans Cedex, France \\ ${ }^{3}$ Istituto dei Sistemi Complessi, CNR, via dei Taurini 19 Roma, Italy and INFN Sezione di “Tor Vergata," Roma, Italy
}

(Received 20 June 2017; published 5 February 2018)

\begin{abstract}
We present an efficient point-particle approach to simulate reaction-diffusion processes of spherical absorbing particles in the diffusion-limited regime, as simple models of cellular uptake. The exact solution for a single absorber is used to calibrate the method, linking the numerical parameters to the physical particle radius and uptake rate. We study the configurations of multiple absorbers of increasing complexity to examine the performance of the method by comparing our simulations with available exact analytical or numerical results. We demonstrate the potential of the method to resolve the complex diffusive interactions, here quantified by the Sherwood number, measuring the uptake rate in terms of that of isolated absorbers. We implement the method in a pseudospectral solver that can be generalized to include fluid motion and fluid-particle interactions. As a test case of the presence of a flow, we consider the uptake rate by a particle in a linear shear flow. Overall, our method represents a powerful and flexible computational tool that can be employed to investigate many complex situations in biology, chemistry, and related sciences.
\end{abstract}

DOI: 10.1103/PhysRevE.97.023301

\section{INTRODUCTION}

Reaction diffusion processes are ubiquitous in many contexts ranging from physics and chemistry to engineering [1]. They are also key in biology, where they control enzyme catalysis, antigen-antibody encounter, fluorescence quenching, and cellular nutrient uptake [2-4], which serves as the main motivation for this paper. Nutrient uptake typically takes place in a fluid: flow can therefore modify the reaction rates $[5,6]$. This is particularly relevant to unicellular organisms, as the presence of advection (possibly in combination with motility) modifies the nutrient concentration field and thus the uptake rate [3]. In recent years, the interest toward the problem of chemical reactions involving self-propelled bodies in a flow has increased also due to the technological advancements in chemically powered micro- and nanoswimmers [7-9].

Here we focus on the widespread diffusion-limited reactions [1], corresponding to the limit of reactions whose chemical step proceeds much faster than the diffusive transfer of the components. Cellular uptake by a spherical cell of radius $R$ can be approximated [2] by imposing perfect absorbing conditions at the particle surface (i.e., vanishing concentration field $\rho$ on the sphere's surface). For an isolated spherical cell of radius $R$ much larger than the nutrient's size, the stationary reaction (or uptake) rate is given by the Smoluchowski formula [10] $\kappa_{s}=4 \pi D R \rho_{\infty}$, where $D$ is the diffusion constant of the nutrient field and $\rho_{\infty}$ is the concentration at infinity. When many absorbing cells are present, diffusive interactions come into play [11-15]. This problem of nutrient shielding becomes even more complex in the presence of a flow that transports the reactant and/or when the cells move autonomously. Other complex situations of biological interest include the effects of concentration fluctuations [16]; of confining, compartmentalization, and active transport of reactants $[17,18]$, such as for many biochemical reactions occurring within the cell; and the complex dynamical organization of the plasma membrane [19], where dynamic clustering [20,21], lipid-raft association $[22,23]$, and interactions with cytoskeletal elements [19] of receptors are central in regulating how ligand binding triggers biochemical signaling cascades [24].

In all these cases, one is interested in quantifying the relative efficiency of the process in terms of the ratio between the total uptake rate and the bare diffusive uptake rate of isolated absorbers and receptors, i.e., the Sherwood number Sh. For instance, $\mathrm{Sh}<1$ is typically an indication of diffusive interactions (i.e., mutual screening of diffusive ligand flux among receptors, leading to destructive interference) $[11,14,15]$, while Sh $>1$ can be obtained when the cell moves relative to the surrounding fluid [3]. Clearly, understanding the adaptations leading to (or induced by) values of Sh differing from 1 is key to deciphering the life strategies of many unicellular organisms [4].

Advancements in this field require experimental, theoretical, and computational work coupling fluid dynamics, ruled by the Navier-Stokes equation, with the reaction-diffusion rules of reactants. In the case of natural or artificial microswimmers, theory and computations must correctly describe particles that are advected by the flow, modify it, and react with the transported concentration fields. This is a formidable challenge, as it requires resolving the dynamics on many scales, particularly when the fluid is turbulent.

In the absence of a flow, several computational methods have been developed, based on the finite-element method $[25,26]$, the molecular-dynamics method $[27,28]$, multipole expansion techniques [29-31], and first-passage Monte Carlo techniques [32-36]. In principle, in this case diffusive interactions among many different boundaries can be accounted for exactly via reexpansion formulas for a wide array of geometries $[37,38]$. Recently, for example, translation addition theorems 
for solid spherical harmonics have been used to compute the reaction rate of diffusion-influenced reactions [15] and to investigate transient heat transfer [39] in the presence of many spherical boundaries. These theoretical treatments have the advantage that in many cases simple analytical estimates can be obtained by truncating the associated multipole expansions. For example, when the majority of boundaries are absorbing, simple monopole approximations have been shown to yield surprisingly accurate results $[11,12,15]$. The case of moving absorbers has also been investigated in [40,41].

Conversely, for the problem of nutrient uptake in the presence of a flow, there are fewer numerical investigations. Recent works have studied the uptake of nutrients by active swimmers in a thin film stirred by their motion [42] and by diatom chains in two-dimensional flow [43]. These studies have generalized the immersed boundaries method (IBM) [44] to account also for the reaction process. The IBM converts the no-slip boundary condition at the body (of the particle or of other structures) into a set of forces applied on the fluid in the neighborhood of the particle surface so as to ensure that the boundary conditions are fulfilled. In the same spirit, the boundary conditions on the nutrient concentration field are imposed in terms of appropriate sinks around the particle [42]. When considering many (possibly swimming) particles in a stirred fluid, potentially turbulent, the above methods become too complex to be used unless limiting the number of particles, which need several grid points to be properly resolved.

In this work, we present a numerical method for computing the diffusion-limited uptake of nutrients by small spherical particles inspired to the force coupling method (FCM), introduced by Maxey and collaborators $[45,46]$. The basic idea of the FCM is to represent each particle by a force distributed over a few grid points. Notwithstanding these limitations, the method is numerically very effective and compares well with analytical [46] and experimental results [47]. We extend the FCM to the transport of nutrients by replacing the absorbing boundary conditions with an effective sink of concentration localized at the particle position (see [48] for a similar approach). This method can be easily implemented in the presence of a flow and also for self-propelled particles. In this work, however, we mainly focus on the diffusive problem and compare the results of the FCM with analytical solutions and with the exact solutions obtained by a multipole expansion method coupled to reexpansion formulas [15]. In this method, the stationary density field is written formally as a sum of as many multipole expansions as there are boundaries (and local spherical frames of reference). Then, translation addition theorems for solid spherical harmonics [38] are used to express the whole density field on each boundary in turn, so that the appropriate local boundary conditions can be imposed as many times as there are boundaries. We also present preliminary results for a single absorber in the presence of a linear shear flow, leaving the study of more complex flows to future investigations.

The material is organized as follows. In Sec. II we present the method, its implementation, and we consider the case of an isolated spherical absorber to explain how the numerical parameter should be calibrated in order to reproduce the Smoluchowski result. Then in Sec. III we show the results of the numerical method in resolving the diffusive interactions between multiple absorbers in different configurations. In particular, we consider two absorbers placed at varying distance. Here we can compare with an exact analytical theory [15], allowing us to discuss the limitations of the method when the particles are too close, or too far apart. After that, we consider triads (triangles) and tetrads (regular tetrahedra) of particles. Then we use the method to study random clusters of absorbing particles, either filling a sphere or a spherical shell, comparing the results with both exact numerical calculations and approximate analytical theories. Finally, we show how the reaction rate is modified in the presence of a linear shear flow, comparing our results with approximate theories developed in [49]. In the final section, we draw our conclusion and describe some possible applications of our method.

\section{THE NUMERICAL METHOD}

We consider a set of $N$ absorbing spherical particles of radius $R_{i}$ at positions $\boldsymbol{X}_{i}(t)(i=1,2, \ldots, N)$. The scalar field $\rho(\boldsymbol{x}, t)$ obeys the diffusion equation with an absorbing boundary condition (i.e., $\rho=0$ ) on the spheres' surface. As discussed in the Introduction, we replace the boundary conditions at the particle surface by a volumetric absorption process of first order localized over regularized $\delta$ functions $f\left(\boldsymbol{x}-\boldsymbol{X}_{i}\right)$ centered on the particle positions. Hence the concentration field obeys the equation

$$
\partial_{t} \rho=D \nabla^{2} \rho-\rho \sum_{i}^{N} \beta_{i} f\left(\boldsymbol{x}-\boldsymbol{X}_{i}\right),
$$

where $\beta_{i}$ is the (constant) volumetric absorption rate of particle $i$. By making $\beta_{i}$ depend on the concentration, the sink term in (1) can mimic the saturable kinetics of Michaelis-Menten-type often used for modeling cellular uptake [43]. In this work, however, we are interested in modeling perfectly absorbing spheres, for which a number of results are at hand. Therefore, we take the absorption rate constant and we have to determine how $\beta_{i}$ is related to the effective radius of the absorbing sphere. We remark that our method can be implemented also in the presence of a flow by adding the advection term in (1), and also in the case of self-propelled particles, including the fluidparticles interactions $[45,46]$.

We integrate the diffusion equation (1) by a standard pseudospectral method in a cubic domain of size $L=2 \pi$ consisting of $M^{3}$ grid points (with $M$ between 64 and 256) with periodic boundary conditions in all directions. Time evolution is computed by using a second-order Runge-Kutta scheme with exact integration of the linear term. The use of periodic boundary conditions makes the problem equivalent to the case of an infinite periodic cubic lattice of sinks, for which the total concentration decays in time [12]. To reach a stationary state, one can add a source term to (1), e.g., by imposing a fixed concentration over a large bounding sphere in the computational domain [14], but this cannot be used in the presence of a flow. Another possibility is to add a homogeneous source term to (1), as was done in [48]. Because here we are mainly interested in benchmarking the numerical method with known results of isolated absorbers in an infinite volume, we add no source terms to the equations, and we perform the simulations in the condition of a slowly decaying nutrient. Nonetheless, by considering a sufficiently 
large domain with respect to the absorber configurations, the effects due to periodicity appear only at long times, and, as we will see, they do not limit the possibility to measure the nutrient uptake in conditions equivalent to the infinite domain.

There are several possibilities to implement the regularized $\delta$ function $f(\boldsymbol{x})$. For instance, for particle-flow interaction a Gaussian function is typically employed $[45,46]$. The Gaussian, however, does not have a compact support and thus is numerically not very convenient. Here, we adopt a computationally more efficient choice inspired to immersed boundary methods [44]. We consider the discretized $\delta$ function $f(\boldsymbol{x})$ as the product of identical one-variable functions $\phi(x)$ rescaled with the mesh size $\delta x=L / M$,

$$
f(x)=\frac{1}{\delta x^{3}} \phi\left(\frac{x}{\delta x}\right) \phi\left(\frac{y}{\delta x}\right) \phi\left(\frac{z}{\delta x}\right),
$$

where $x, y, z$ are Cartesian coordinates. The function $\phi$ is chosen to be symmetric, positive, with a compact support around its center, and normalized. The numerical implementation of (1) requires an evaluation of (2) on a discrete number of points spaced by $\delta x$. A convenient choice of $\phi$, which is normalized independently of the number of support points and of the position of the center relative to the grid (i.e., approximately grid-translational-invariant), is [44]

$$
\phi\left(\frac{x}{\delta x}\right)= \begin{cases}\frac{1}{n}\left[1+\cos \left(\frac{2 \pi x}{n \delta x}\right)\right], & \left|\frac{x}{\delta x}\right| \leqslant \frac{n}{2} \\ 0 & \text { otherwise. }\end{cases}
$$

The particle has a "numerical radius" given by $a=(n / 2) \delta x$, which is in general different from its effective radius $R$, i.e., the radius of the equivalent absorbing sphere, which will (as shown below) depend on both $a$ and $\beta_{i}$. Note that the particle position $\boldsymbol{X}_{i}$ in (1) takes real values in three-dimensional space. Consequently, the smoothed $\delta$ function is centered at any arbitrary position, but the function itself is evaluated only on $n^{3}$ grid points.

The uptake rate $\kappa_{i}$ of particle $i$ can be directly computed from the integral of the sink term in (1),

$$
\kappa_{i}(t)=\int \beta_{i} f\left(\boldsymbol{x}-\boldsymbol{X}_{i}\right) \rho(\boldsymbol{x}, t) d^{3} \boldsymbol{x},
$$

where the integral is numerically evaluated by the sum over the grid points defined in (3). The global uptake rate is then obtained by summing (4) over all the particles, or alternatively measuring the rate of change of the volume averaged concentration $C(t) \equiv\langle\rho\rangle=V^{-1} \int \rho(\boldsymbol{x}, t) d^{3} \boldsymbol{x}$. By integrating (1), it is easy to see that

$$
\frac{d C}{d t}=-\frac{1}{V} \sum_{i=1}^{N} \kappa_{i}(t)
$$

\section{A. Calibration of the numerical method}

In this section, we show how the effective radius $R$ of an absorber depends on $\beta$ and the numerical radius $a$. Toward that end, we perform a set of numerical simulations considering a single absorbing sphere in an initially uniform scalar field, $\rho(\boldsymbol{x}, 0)=\rho_{0}$, for different values of the absorption rate $\beta$. In all simulations, we fixed $n=4$ in (2) (as is customary in the IBM [44]), the scalar diffusivity $D=0.01$, and $\rho_{0}=1$.

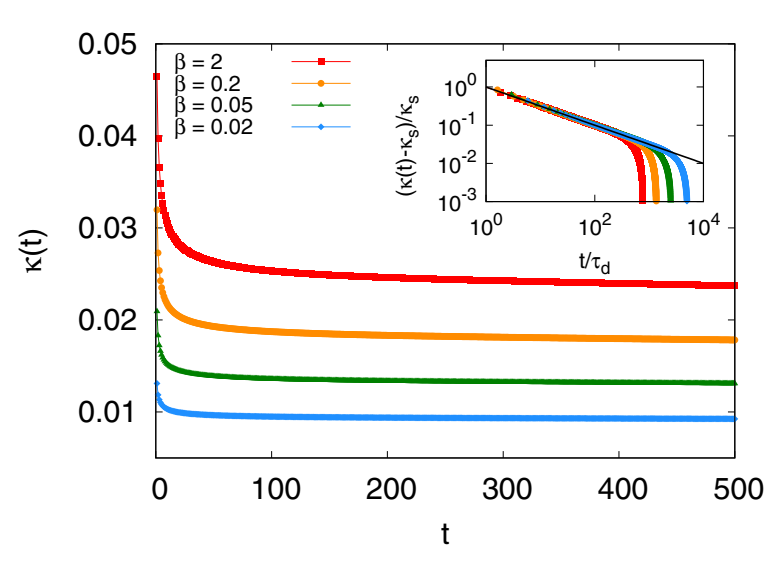

FIG. 1. Time evolution of the uptake rate $\kappa(t)$ for different values of $\beta$, from top to bottom: 2 (red), $2 \times 10^{-1}$ (orange), $5 \times 10^{-2}$ (green), and $2 \times 10^{-2}$ (cyan). Inset: $\left[\kappa(t)-\kappa_{s}\right] / \kappa_{s}$ vs $\tau=t / \tau_{D}\left[\tau_{D}=\right.$ $R^{2} /(\pi D)$ with $R$ obtained from the fit based on Eq. (6)]. For short times, all the curves collapse on the line $1 / \sqrt{\tau}$ (black line) as predicted by (6). Simulations have been performed with resolution $M=64$.

The effective radius can be obtained comparing the absorbing rate with the Smoluchowski result. More precisely, since our simulations are time-dependent, as explained above, one needs to compare the time evolution of the uptake rate (4) with the Smoluchowski solution of the time-dependent diffusive problem (see Appendix A):

$$
\kappa_{s}(t)=4 \pi D R \rho_{\infty}\left(1+\frac{R}{\sqrt{\pi D t}}\right) .
$$

We use the same symbol for both the time-dependent and the steady solution, for the latter $\kappa_{s}=4 \pi D R \rho_{\infty}$, obtained from (6) when $t \rightarrow \infty$, the time dependence is omitted.

Figure 1 shows the evolution of the uptake rate $\kappa(t)$, computed from (4), as a function of time for different values of $\beta$. Two regimes are observed: at the beginning the diffusive regime described by (6) is well evident (see the inset), while for longer times a slower decay due to the boundedness of the domain sets in. By fitting $\kappa(t)$ with the expression (6) in the first regime, one obtains two independent estimates of $R$ (from the constant term and from the time-dependent term). For all values of $\beta$, the two measures give the same value of $R$ within $2 \%$ of error.

The result of the calibration for the effective radius $R$ is shown in Fig. 2 for different resolutions $M$. For small $\beta$, the effective radius $R$ is proportional to the absorption rate. For large $\beta, R$ saturates to $a=(n / 2) \delta x$, which depends on the resolution as $n$ is fixed and the mesh size changes as $\delta x=$ $L / M$.

To rationalize this behavior and eventually find an analytical fitting expression for $R$, we resorted to a crude approximation for the regularized $\delta$ function assuming a spherical sink function of radius $a$, in polar coordinates $f(r)=\Theta(a-r) / V_{a}$, where $\Theta$ is the Heaviside step function, $r$ is the distance from the sphere center, and $V_{a}=4 \pi a^{3} / 3$ is the sphere volume. With this form for $f(r)$, the stationary solution of (1) in infinite space is easily solved (see Appendix B). The analytic expression of the uptake rate [see Eq. (B5)], when compared with the 


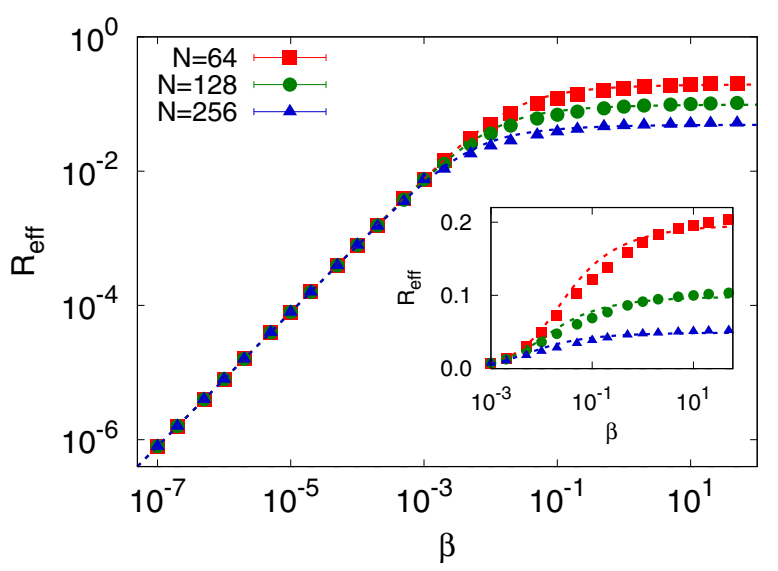

FIG. 2. Effective radius $R$ obtained from $\kappa(t)$ fitted with (6) vs $\beta$ for different resolutions as labeled. The dashed curves display Eq. (7) with $a=(n / 2)(L / M)$. Inset: zoom in the range $\beta>10^{-3}$.

Smoluchowski rate, yields

$$
R=a-\sqrt{\frac{D V_{a}}{\beta}} \tanh \left(a \sqrt{\frac{\beta}{D V_{a}}}\right),
$$

which shows a remarkable (within 10\%) agreement with the effective radius obtained with the fitting procedure (see Fig. 2). Thus Eq. (7) can be used as the calibrating function. Notice that for small $\beta$, Eq. (7) yields $R=\beta /(4 \pi D)$, which is the result one would obtain by replacing $f$ with a true $\delta$ function in (4), while $R \rightarrow a$ for large $\beta$. Typically, in our simulations we fixed $\beta=D=0.01$, which leads to an effective radius $R \lesssim a$.

It is worth remarking that, as in the case of the forcecoupling method for fluid-particle interaction [45,46], the diffusive boundary layer is not well resolved at the scale of the regularization. This is apparent in Fig. 3, which displays the profile of the scalar field as a function of the distance from the particle. The analytical expression obtained from Eq. (A2) agrees well with the numerical result only for $r>2 a$,

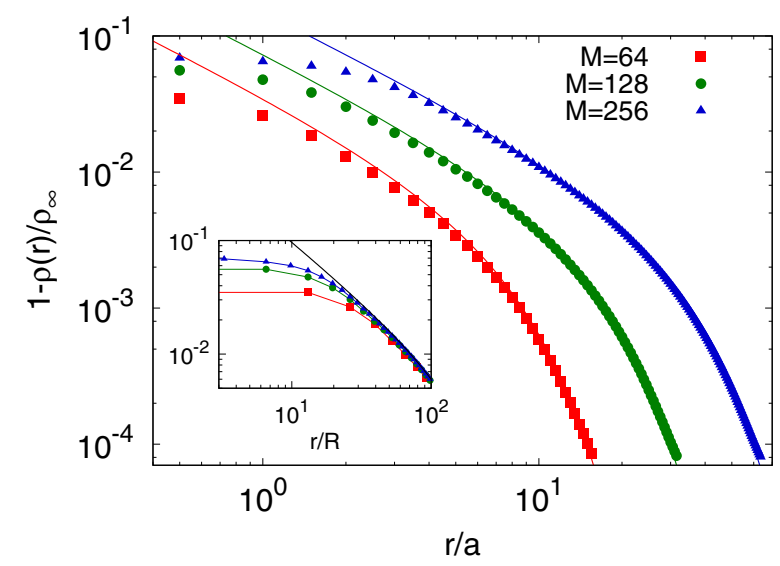

FIG. 3. Radial profile of the concentration field $\rho(\boldsymbol{x}, t)$ obtained from simulations at time $t=5 \times 10^{4} \tau_{D}$ as a function of $r / a$ for different resolutions compared with the theoretical prediction (A2) (solid lines). Inset: the same quantity as a function of the resolutionindependent coordinate $r / R$. Parameters: $D=10^{-2}, \beta=10^{-3}$.

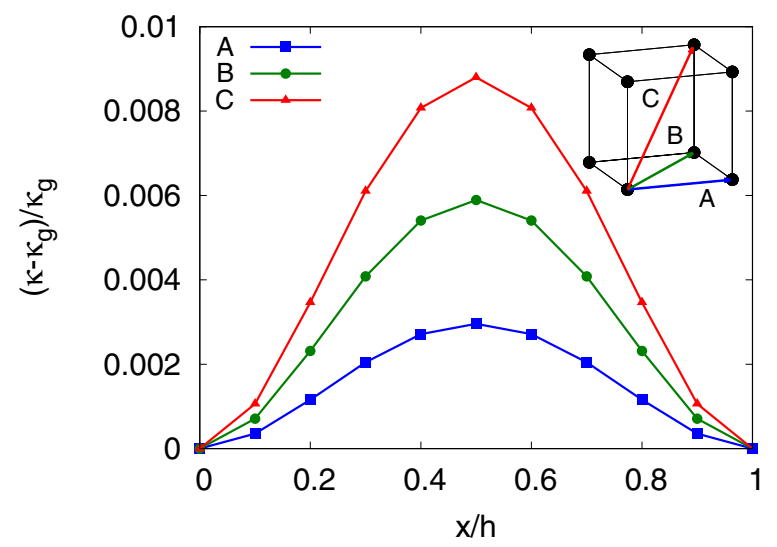

FIG. 4. Relative error on the uptake rates depending on the position of the particle within the lattice unit. We vary the distance $x$ from a chosen grid point, along three different paths (shown in the inset) of size $h$, from bottom to top: side of the grid unit (blue, $h=\delta x$ ), face diagonal (green, $h=\sqrt{2} \delta x$ ), and cube diagonal (red, $h=\sqrt{3} \delta x$ ). On the $y$ axis we show the discrepancy between the off-grid uptake rate $\kappa$ and the one measured on a grid point $\kappa_{g}$. Along each path, the discrepancy is maximal at the farthest position from the grid. Simulations refer to the resolution $M=64$.

and therefore the accuracy of the method increases with the resolution, as shown in the inset of Fig. 3. This, as we will see in the next section, has some consequences on the ability of the method to resolve the diffusive interactions of close particles.

Finally, in Fig. 4, we assess possible systematic errors coming from varying the position of the particle in the grid, i.e., errors due to the use of the regularized $\delta$ function (2). We measured the relative error on the uptake rate varying the position of the particle along the side $\delta x$ of a lattice unit, along the face diagonal and along the cube diagonal (see the inset). The largest variation observed was less than $1 \%$ for a regularization on $n^{3}=64$ grid points. Although it is not shown here, we also checked that such variation decreases with the resolution (down to $0.3 \%$ for $M=256$ ).

\section{CONFIGURATIONS WITH MULTIPLE ABSORBERS}

In this section, we consider $N$ static absorbing particles, arranged in configurations of increasing complexity from regular to random, with the aim of testing the reliability and precision of our method. For the sake of simplicity, we discuss only cases in which all particles have the same radius, i.e., $\beta_{i}=\beta$ in Eq. (1). All simulations are initialized with a uniform scalar field, $\rho(\boldsymbol{x}, 0)=\rho_{0}=1$. The asymptotic uptake rates are evaluated as discussed in Sec. II A by fitting $\kappa(t)$ with (6) on each particle. Indeed, it can be shown that the functional form (6) holds also in the case of multiple sinks $[11,12]$.

We compare the numerically obtained rates with those obtained from a numerical multipole expansion algorithm [15]. When available, we also compare our results with analytical exact or approximate expressions. The main aim of this study is the validation of our method in resolving the diffusive interaction, quantified by the Sherwood number Sh defined as the total absorption rate normalized with that of $N$ isolated 


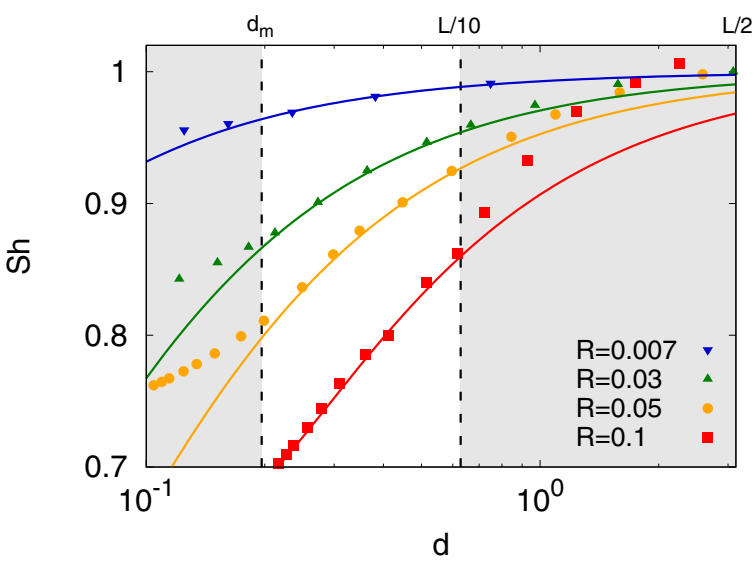

FIG. 5. Sh as a function of the distance $d$ between two spherical absorbers of radius $R$, varied as in the legend. Solid curves represent the exact result (9). The gray shaded regions highlight the range of distances where the numerically obtained Sh becomes sensibly different from the exact value; see the text for discussion. The simulations have been performed with $M^{3}=64^{3}$ grid points.

absorbers,

$$
\mathrm{Sh}=\frac{\kappa_{\mathrm{tot}}}{N \kappa_{s}} .
$$

In the final subsection, we shall consider the case of a single absorber in the presence of a linear shear flow and study the Sherwood number as a function of the Peclet number, quantifying the ratio between advective over diffusive transport.

\section{A. Pairs of absorbers $(N=2)$}

The case of two spherical absorbers of radius $R$ separated by a distance $d$ is one of the few examples of the diffusive interaction problem that can be solved exactly. After choosing bi-spherical coordinates, the Laplace equation becomes separable [38] and the total absorption rate depends on the relative distance $x=d / 2 R$ as $[50,51]$

$$
\mathrm{Sh}=\sqrt{x^{2}-1} \sum_{n=0}^{\infty} \frac{2}{1+\left(x+\sqrt{x^{2}-1}\right)^{2 n+1}} .
$$

In the limit of well-separated absorbers, $x \rightarrow \infty$, Eq. (9) yields the noninteracting result $\mathrm{Sh}=1$ (i.e., both spheres absorb the nutrient at the Smoluchowski rate as if they were isolated). Notice that, already for $x \gtrsim 2$ the first correction given by the monopole contribution,

$$
\mathrm{Sh}=\frac{2 x}{2 x+1}=\frac{d}{d+R},
$$

is a very good approximation of (9). In the limit of two spheres in contact, $x=1$, Eq. (9) gives the maximum interference, with $\mathrm{Sh}=\ln (2)$.

In Fig. 5, we show the numerically computed Sherwood number as a function of the pair distance $d$ for different choices of the particle effective radius $R$ at fixed resolution. The numerical results are directly compared with the exact value obtained from (9). A very good agreement between numerical and theoretical values is observed for distances

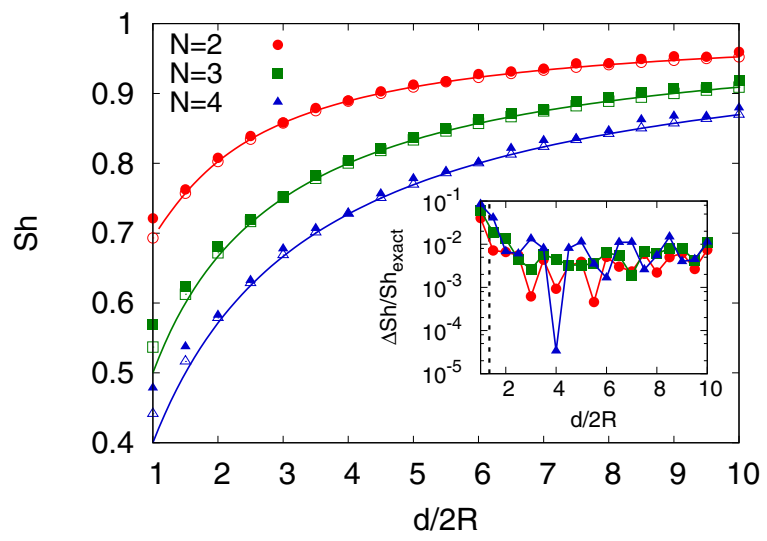

FIG. 6. Sh for configurations of $N$ equidistant absorbers as a function of the dimensionless distance $x=d / 2 R$ for $N=2,3,4$ as in the legend. Filled symbols are the results from the numerical simulations, and open symbols are the exact results obtained with the method of Ref. [15]. Lines represent the monopole approximation Eq. (12). The inset shows the relative error with respect to the exact solutions. The vertical dashed line is positioned at $x_{m}=d_{m} / 2 R$, marking the condition for well-resolved simulations. On the left of the threshold line the error rises to 4-8\%, while for the other distances it is always close to $1 \%$. The resolution used is $M=128$.

larger than $d_{m} \approx a=(n / 2) \delta x$, and about $1 / 10-1 / 8$ smaller than the domain size $L=2 \pi$.

The discrepancies at small distances are due to the fact that the method does not resolve the particle surface: the numerical radius, $a$, imposed by the regularized $\delta$ function turns out to be the limiting distance for resolving the pair diffusive interactions (see also Fig. 3 and related discussion), regardless of the effective radius of the particles. To reduce $d_{m}$ the only possibility is thus to increase the resolution.

The large-distance discrepancies are due to the periodicity of the simulation domain. Since the diffusive interactions are long-ranged [they decay with the inverse of the distance from the absorbers; see Eq. (A2)], when $d$ increases the particles start to interact not only with each other but also with their periodic images, leading to an increase of the total uptake rate (i.e., Sh becomes larger than predicted for a pair of absorbers at the same distance in infinite space). This effect tends to increase with the effective radius $R$ of the particle as the diffusive interaction increases with $R$ [see Eq. (A2)]. We emphasize that this effect cannot be modified by changing the resolution, but it requires working with different boundary conditions.

Summarizing, the above results show that provided the particles are at distances $(n / 2) \delta x=d_{m}<d<d_{M} \approx L / 10-$ $L / 8$, the numerical method works quite well. Figure 6 shows $\mathrm{Sh}$ as a function of the rescaled distance $x=d / 2 R$ together with the exact result and the monopole approximation. As is clear from the inset, in the whole range of $x$ the numerical values are within $2 \%$ from the exact result with larger deviations, $\sim 4 \%$, when $x \rightarrow 1$ corresponding to distances $d \approx d_{m}$. In the following, we shall exploit these results when studying more complex arrangements of absorbing particles, making sure that the particles stay at distances within the range of scales for which the method works well. 


\section{B. Regular triangles $(N=3)$ and tetrahedra $(N=4)$}

We now consider regular arrangements of $N=3$ and 4 particles at varying distances. From the theoretical side, the monopole expression (10) can be easily extended to the case of $N>2$ absorbers. Within the monopole approximation, one can write the set of linear equations for the uptake rate $\kappa_{i}$ of the $i$ th absorber [11,12],

$$
\kappa_{i}=\kappa_{s}^{i}-\sum_{j \neq i}^{N} \epsilon_{i j} \kappa_{j}, \quad i=1, \ldots, N,
$$

where $\epsilon_{i j}=R_{i} / d_{i j}, R_{i}$ is the radius of the $i$ th sphere, $d_{i j}$ is the distance between the $i$ th and $j$ th sink, and $\kappa_{s}^{i}=4 \pi D R_{i} \rho_{\infty}$. The case considered here is $R_{i}=R$ and $d_{i j}=d$ for $N=3,4$. In this limit, the total Sherwood number, in the monopole approximation, is given by $(x=d / 2 R)$

$$
\mathrm{Sh}=\frac{2 x}{2 x+(N-1)} .
$$

The numerical results are shown in Fig. 6, together with the monopole expression (12) and the exact results computed by using the approach described in Ref. [15]. The limit $x=1$ corresponds to the minimal distance at which the spheres are at contact and therefore to the maximum diffusive interaction. From a numerical standpoint, with our choice of $\beta$, this limit corresponds to $d \approx d_{m}$. The discrepancy between the numerical simulations and the exact results is maximal here, between $4 \%$ and $8 \%$ (see the inset), increasing with $N$ as intuitively expected. At larger distances, the exact values are recovered within $\lesssim 2 \%$. It is remarkable that the interaction is still observed for $x \simeq 10$, as a consequence of the longrange nature of the diffusive interactions. Notice that, to reach $x=10$ without violating the constraint imposed by the periodic boundary conditions (cf. Fig. 5), we have varied $x$ also changing the effective radius. We finally remark that as soon as $x>2$, the monopole approximation practically coincides with the exact result.

\section{Deformed triangles $N=3$}

We considered also the case of three spheres of radius $R$ at the vertices of irregular triangles, as sketched in the inset of the top panel in Fig. 7. A practical way to construct the triangle is the following: We fix the distance between particles 1 and 2 to be $d_{12}=X d_{m}$ with $X>1$. Let us denote with $\theta$ the angle between the segments 12 and 13. We keep this angle fixed and vary the distance $d_{13}=d$, requiring $d_{i j} \geqslant d_{m}$ for $i \neq j=$ $1,2,3$, which implies a minimal angle $\theta_{c}=\arcsin (1 / X)$. Here we fix $X=5.5$ so that $\theta_{c} \sim 14.35$. In the simulations, we used $\theta=15^{\circ}, 20^{\circ}, 30^{\circ}, 60^{\circ}$ and varied $d$ in the range $\in[2 R, 20 R]$. As for the parameters of the simulation, we fix $D=\beta=0.01$ in such a way that the radius of the spheres $R=0.036$.

The solution of such configurations in the monopole approximation using Eq. (11) is given by

$$
\mathrm{Sh}=\frac{1}{3}+\frac{2}{3} \frac{(A-1)(B-1)(C-1)}{A^{2}+B^{2}+C^{2}-2 A B C-1},
$$

where $A=R / d_{12}$ with $d_{12}$ fixed, $B=R / d_{13}$, and $C=R / d_{23}$ with $d_{23}=\sqrt{d_{12}^{2}+d_{13}^{2}-2 d_{12} d_{23} \cos \theta}$.
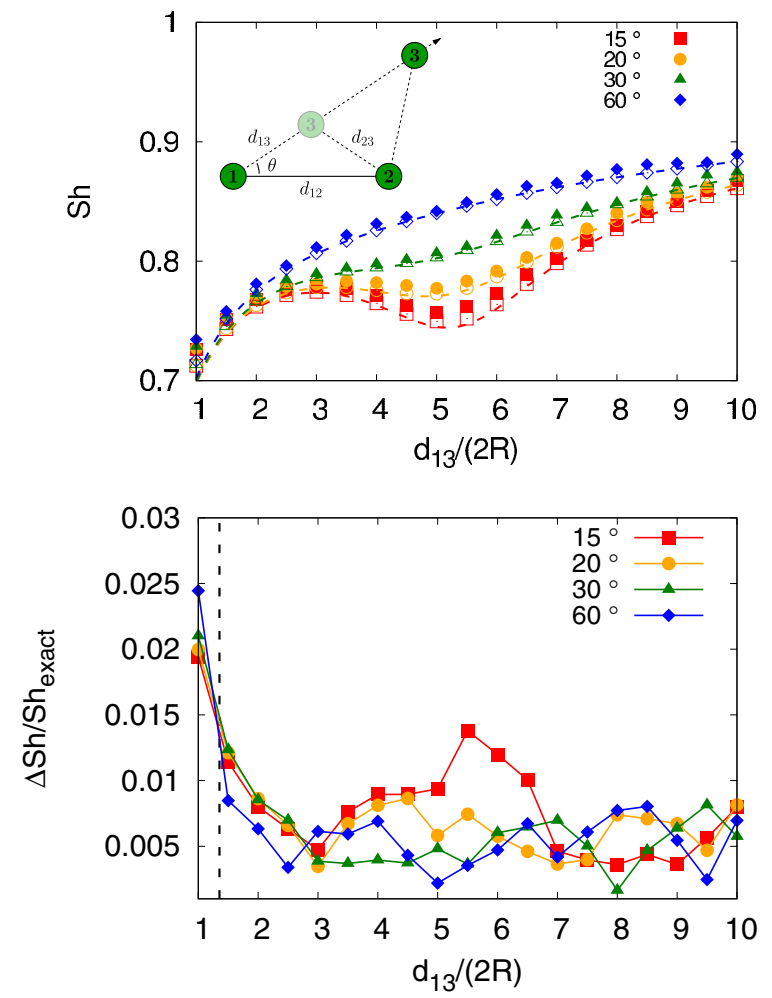

FIG. 7. Top: Sh for different configurations of deformed triangles with $\theta=15^{\circ}, 20^{\circ}, 30^{\circ}, 60^{\circ}$ as a function of the distance $d_{13}$ normalized by the sphere diameter $2 R$. The minimum of Sh corresponds to the configuration of minimal distance $d_{23}$ (filled symbols from the numerical simulations, open symbols obtained with the method of Ref. [15]). Inset: sketch of the deformed triangles. Bottom: relative error of the simulations with respect to the exact results obtained with the method of Ref. [15]. The dashed line represents the critical distance $x_{m}=d_{m} /(2 R)$ below which the numerical accuracy is decreased as discussed in Sec. III A.

In Fig. 7 we plot the total Sherwood number of the triadic system as a function of the distance $d_{13}$ normalized by the diameter of the absorber $2 R$. Our simulations are compared with the exact results obtained following the method of Ref. [15]. We also compare the results with the monopole approximation (13). The minimal uptake is obtained in the configuration with minimum distance $d_{23}$, which maximizes the diffusive interactions. As shown in bottom panel, the error is within $2 \%$ but for configurations with $d_{13} \approx d_{m}$, as expected from previous discussions. We conclude by noticing that when particle 3 is moved far away from the pair, we recover the asymptote (not shown) given by the uptake of a single sphere and the contribution of the pair alone, which with our choice is $\mathrm{Sh} \simeq 0.94$.

\section{Random spherical cluster}

In this section, we consider the case of a cluster of absorbers. One important motivation comes from biology in the case of colonies of micro-organisms. In this case, one is interested in understanding how diffusive interactions, which cause nutrient shielding for cells in the cluster interior, deplete the growth of the colony [14]. 

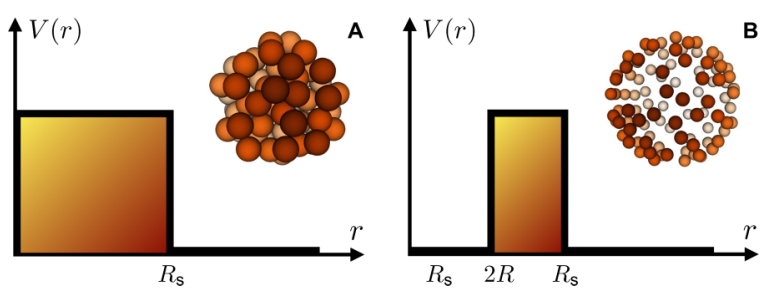

FIG. 8. Sketch of the cluster configurations: (a) spherical cluster; (b) spherical shell cluster. The graphs also show the potential used to develop the effective-medium approximation.

Specifically, we consider a spherical cluster of absorbers, i.e., a sphere of radius $R_{s}$, centered at the origin, comprising $N$ spherical absorbers, with the same radius $R$, randomly arranged in its interior avoiding geometrical overlaps [see Fig. 8 (a)]. In this case, it is possible to have an analytical estimation of the nutrient uptake by introducing an effectivemedium approximation [14].

The basic idea of the method is to introduce an effective concentration field $\psi(\boldsymbol{x}, t)=\langle\rho(\boldsymbol{x}, t)\rangle$, where the angular brackets denote an ensemble average over the possible random position of the absorbing particles. By averaging both sides of Eq. (1) and assuming stationarity, one has

$$
\begin{aligned}
D \nabla^{2} \psi & =\left\langle\sum_{i=1}^{N} \beta_{i} \rho(\boldsymbol{x}) \delta\left(\boldsymbol{x}-\boldsymbol{X}_{i}\right)\right\rangle \\
& \simeq \int \chi\left(\boldsymbol{x}-\boldsymbol{x}^{\prime}\right) \psi\left(\boldsymbol{x}^{\prime}\right) d \boldsymbol{x}^{\prime}
\end{aligned}
$$

where $\chi(x)$ is a linear-response function describing the deformation of the concentration field induced by the absorption [14]. The linear-response approximation is only valid for sufficiently small concentration field deformations (dilute clusters) and away from the cluster edges. Fourier-transforming Eq. (14), one obtains the equation $-D q^{2} \hat{\psi}(\boldsymbol{q})=\hat{\chi}(\boldsymbol{q}) \hat{\psi}(\boldsymbol{q})$. Since, on average, the cells are isotropically distributed, $\chi(\boldsymbol{q})$ can only depend on $q=|\boldsymbol{q}|$. Expanding $\hat{\chi}$ around $q=0$ and truncating at zeroth order, i.e., $\hat{\chi}(0)=k$, provides the desired mean-field approximation. Hence the configurationally averaged nutrient concentration obeys the equation $D \nabla^{2} \psi-$ $k \psi=0$ valid within the sphere of radius $R_{s}$ delimiting the cluster, outside $D \nabla^{2} \psi=0$; this is merely the equation we already solved to determine the calibrating function (7) (see Appendix B). In the above expression, $k$ represents an effective absorption rate within the sphere in the macroscopic description. The truncation at zeroth order works reasonably well for dilute clusters, and in this limit $k=4 \pi D R N / V_{s}=3 D \phi / R^{2}$, where $\phi=N R^{3} / R_{s}^{3}=N / \alpha^{3}$ is the volume fraction (with $\alpha=R_{s} / R$ ). The cluster is thus approximated as a unique sink with penetrable walls. We can directly use Eq. (B5) to express the total uptake rate

$$
\kappa_{\mathrm{tot}}=4 \pi D \psi_{\infty}\left[R_{s}-\xi \tanh \left(\frac{R_{s}}{\xi}\right)\right],
$$

$\xi=\sqrt{D / k}=R / \sqrt{3 \phi}$ being the penetration length.
The Sherwood number is defined as $\mathrm{Sh}=\kappa_{\mathrm{tot}} / N \kappa_{s}$, so using $\kappa_{s}=4 \pi D R \psi_{\infty}$ and replacing $N=\alpha^{3} \phi$, from (15) we obtain

$$
\operatorname{Sh}(\phi)=\frac{1}{\alpha^{2} \phi}\left[1-\frac{1}{\alpha \sqrt{3 \phi}} \tanh (\alpha \sqrt{3 \phi})\right] .
$$

Let us now consider the local uptake rate of a cell within the cluster. We denote with $\kappa_{i}$ the uptake rate of the $i$ th particle, and we identify its position in the cluster by its distance $r_{i}$ from the center. We can then introduce the average uptake rate $\kappa(r)=\left\langle\kappa_{i} \mid r_{i}=r\right\rangle$, where the angular brackets indicate the ensemble average over different configurations. The Sherwood number of a cell at a distance $r$ from the center of the cluster will then be $\operatorname{Sh}(r)=\kappa(r) / \kappa_{s}$. By definition, the total uptake rate of the cluster is given by $\kappa_{\text {tot }}=\left\langle\sum_{i}^{N} \kappa_{i}\right\rangle$, while the total flux absorbed by the particles contained in a smaller sphere of radius $r$ is given by

$$
4 \pi D r^{2} \frac{d \psi}{d r}=N \int_{0}^{r} \kappa\left(r^{\prime}\right) p\left(r^{\prime}\right) d r^{\prime},
$$

where $p(r) d r=V_{s}^{-1} 4 \pi r^{2} \theta\left(R_{s}-r\right) d r$ is the probability to find a particle at a certain radial position. By taking the derivative of expression (17), one gets

$$
\operatorname{Sh}(r)=\frac{\xi^{2}}{\psi_{\infty} r^{2}} \frac{d}{d r}\left(r^{2} \frac{d}{d r} \psi\right) .
$$

By noting that $\xi^{2} \nabla^{2} \psi=\psi$, it is easy to see that $\operatorname{Sh}(r)=$ $\psi(r) / \psi_{\infty}$, i.e., the local uptake rate $\kappa(r)$ is proportional to the averaged concentration profile $\psi(r)$.

We turn now to the numerical results. We considered a random distribution of particles in a spherical cluster of radius $R_{s}=L / 8=\pi / 4$ so as to minimize the effects due to the periodic boundary conditions (cf. Fig. 5). As for the absorbers, we considered $N=20,50,100,150$ spheres with effective radius $R \sim 0.036$ so that the (nominal) volume fraction is in the range $\phi=2 \times 10^{-3}-10^{-2}$, that is, small enough for the effective-medium approximation to be accurate. Particles are placed uniformly within the sphere volume, ensuring that they stay at distances larger than $d_{m} \approx(n / 2) \delta x=(n / M) \pi$ to reduce the errors due to poor resolution of the diffusive interaction at short distances (cf. Fig. 5). For each $N$, we considered from 5 to 10 different configurations to perform ensemble averages and thus to compare with the effective field approximation. The same configurations have been used to compute the exact solution with the method of Ref. [15].

Figure 9 shows the average density profile $\psi(r)=\langle\rho(r)\rangle$ compared with the theoretical prediction given by the effective-medium approximation (B4). The agreement is remarkably good.

From the simulations, we can extract the uptake rate of each particle using the standard fitting procedure, which involves the temporal evolution of the uptake rate at intermediate times (see Sec. IIA). By averaging over particles and different configurations, one obtains a measure of the total uptake rate of the cluster. Alternatively, one can extract the uptake rate of the cluster directly from the concentration field $\rho$. The radial profile can be compared with the theoretical prediction (B4) to extract the parameters of interest. From the inner solution, it is possible to extrapolate the penetration length $\xi$ shown in the inset of Fig. 9 with the scaling $R / \sqrt{3 \phi}$. By fitting the behavior 


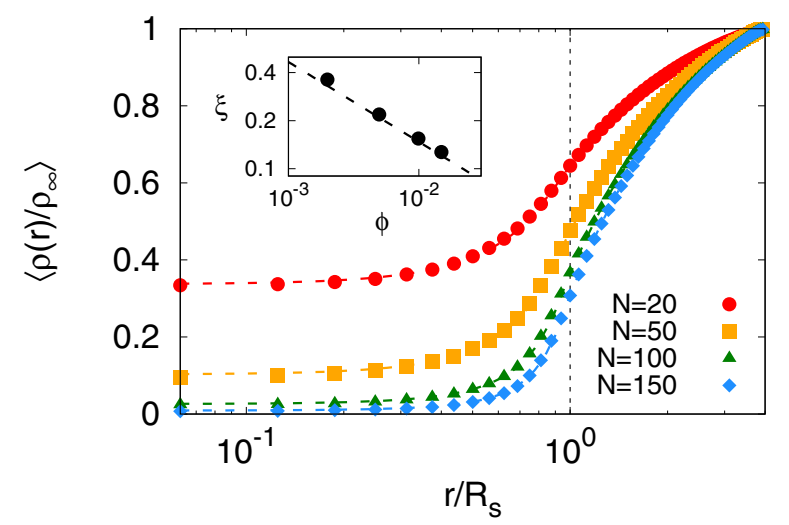

FIG. 9. Average radial concentration profile $\langle\rho(r)\rangle$ vs $r / R_{s}$ for clusters with $N=20,50,100,150$ particles as labeled. Numerical data (symbols) are compared with the effective-medium solution given by Eq. (B4) (dashed curves). Inset: penetration length $\xi$ obtained from the fit of the radial profile (filled circles) and theoretical prediction given by $\xi=R / \sqrt{3 \phi}$ [52] (dashed line). The average is performed over 5-20 independent realizations. The numerical resolution is $M=128$.

in the outer region, we have an alternative estimate of the total Sherwood number.

In Fig. 10 we plot the individual Sherwood number $\mathrm{Sh}_{i}=$ $\kappa_{i} / \kappa_{s}$ as a function of the radial distance of particles in the cluster, compared with the values obtained from the exact numerical solution. The relative difference is below $8 \%$, as shown in the inset, and is larger in the interior of the cluster, due to the accumulation of errors on the concentration density due to the outer absorbers. In Fig. 10 we also plot the theoretical prediction (18).

Finally, in Fig. 11 we show that also the total Sherwood number compares very well with the theoretical prediction (16) and the exact computation (with error within 10\%).

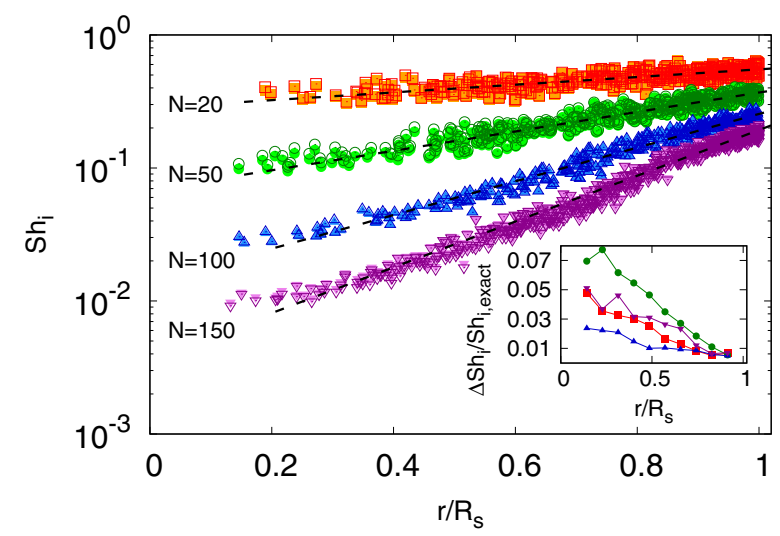

FIG. 10. Individual Sherwood number $\mathrm{Sh}_{i}=\kappa_{i} / \kappa_{s}$ for absorbers in a spherical cluster with $N$ particles as labeled. For each $N, 5-20$ independent realizations are considered. Filled symbols denote the numerical results obtained by our numerical method, while empty symbols are those obtained with the exact numerical computation described in Ref. [15]. Dashed lines represent the prediction (18). Inset: relative errors of the uptake computed as the average absolute distance of the two numerical methods, plotted as a function of the radial coordinate.

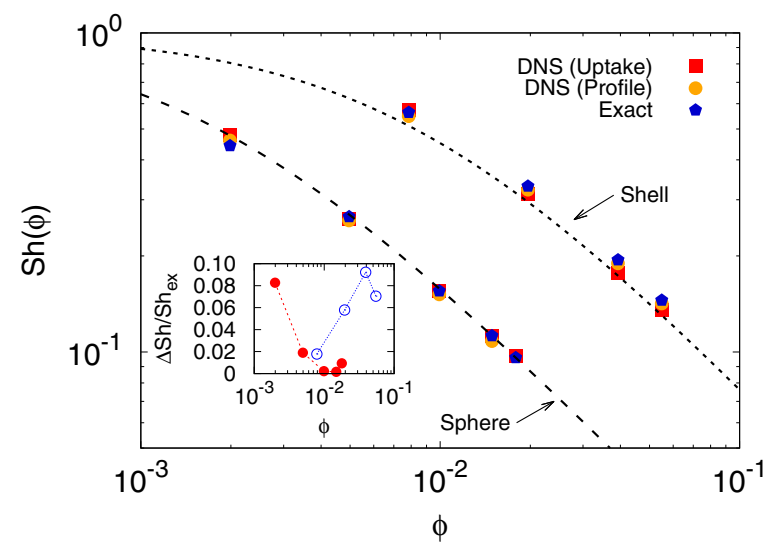

FIG. 11. Total normalized Sherwood number $\mathrm{Sh}=\kappa / N \kappa_{s}$ for the spherical cluster and spherical shell as a function of the volume fraction $\phi$, averaged over 5-10 configurations and obtained by fitting the instantaneous uptake (red squares) or fitting the outer solution of the radial concentration profile (orange circles). Simulations are compared with exact results calculated using the method described in Ref. [15] (blue pentagons). Inset: relative error between simulations and exact results for the spherical cluster (filled circles) and for the spherical shell (empty circles).

\section{E. Spherical shell cluster}

In this section, we study a generalization of the spherical cluster, considering absorbers with their centers at a fixed distance from the origin of the sphere of radius $R_{S}$ [see Fig. 8(b)]. This kind of configuration is encountered in nature. For example, Volvox is a colonial alga forming spherical colonies with a $1 \mathrm{~mm}$ diam. It is usually 100 times larger than the single cell forming it, and it contains up to $5 \times 10^{4}$ cells organized as a monolayer of flagellated cells on the sphere surface [53].

Following the same idea used for developing the effectivemedium approximation of a spherical cluster, one can develop an analytical description for spherical shell clusters. In particular, after averaging over the absorber configurations and performing the expansion of the response function, we end up with the equation

$$
D \nabla^{2} \psi=k\left[\Theta\left(R_{s}-|\boldsymbol{r}|\right)+\Theta\left(|\boldsymbol{r}|-\left(R_{s}-2 R\right)\right)\right] \psi,
$$

where $\Theta$ is the Heaviside step function. The above equation must be solved with the boundary condition $\psi(r \rightarrow \infty)=\psi_{\infty}$ and where again $k=3 D \phi / R^{2}$ in the dilute limit. Now the volume fraction is given by $\phi=N V_{p} / V_{s}$ with $V_{p}=4 \pi R^{3} / 3$ the volume of the single absorber and $V_{s}$ the volume of the shell between $R_{s}-2 R$ and $R_{s}$. The solution of Eq. (19) is detailed in Appendix C.

Similarly to the previous section, introducing $\alpha=R_{S} / R$ and $N=\phi V_{s} / V_{p}=2 \phi\left(3 \alpha^{2}-6 \alpha+4\right)$, and using the expression for the total uptake rate Eq. (C2), after some algebra, the total Sherwood number can be expressed as

$$
\operatorname{Sh}(\phi)=\frac{\frac{1}{2}[\alpha(\alpha-2) \sqrt{3 \phi}-1 / \sqrt{3 \phi}] \tanh (2 \sqrt{3 \phi})+1}{\phi\left(3 \alpha^{2}-6 \alpha+4\right)[(\alpha-2) \sqrt{3 \phi} \tanh (2 \sqrt{3 \phi})+1]}
$$




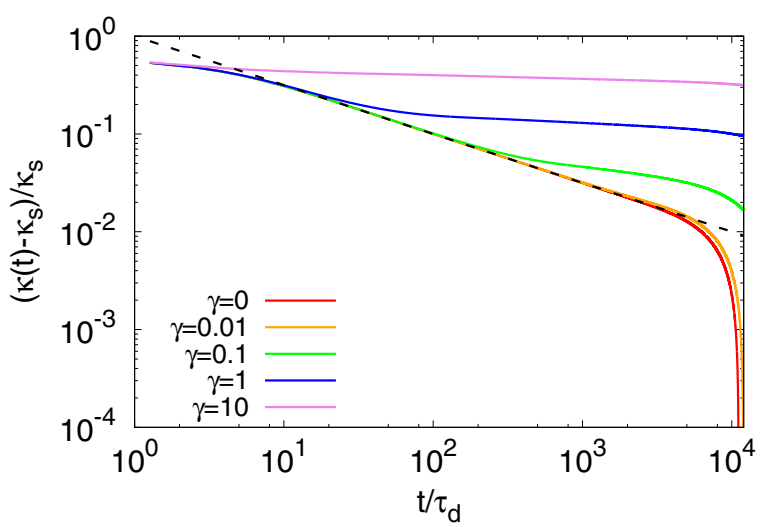

FIG. 12. Time evolution of the uptake rate $\kappa(t)$ rescaled by the diffusive uptake rate $\kappa_{s}$ for different values of $\gamma$ (decreasing from top to bottom) at fixed particle radius $R=0.05(\beta=D=0.01)$. Resolution $M=64$.

In Fig. 11, the total Sherwood number is compared with the theoretical prediction (20); the agreement is good within $10 \%$. It is also interesting to note that the present configuration in spherical shell-like geometry enhances the uptake rates per cell and the total uptake rates of the cluster with respect to the configurations of bulk spherical clusters. Therefore, it can represent an efficient strategy to maximize the uptake rate.

\section{SPHERICAL ABSORBER IN A LINEAR SHEAR FLOW}

To complete our test of the method in the presence of a flow, we consider a single (nonrotating) absorber of radius $R$ placed in the position of zero velocity (so that it does not move) of a linear shear, $\boldsymbol{u}=\gamma(y, 0,0)$, for which analytical results are available $[3,5]$. The nutrient evolves according to Eq. (1) with the addition of the advection term:

$$
\partial_{t} \rho+\boldsymbol{u} \cdot \nabla \rho=D \nabla^{2} \rho-\rho \sum_{i}^{N} \beta_{i} f\left(\boldsymbol{x}-\boldsymbol{X}_{i}\right) .
$$

Analytical results predicts that for small Peclet numbers [54], $\mathrm{Pe}=\gamma R^{2} / D$, the Sherwood number behaves as $[3,49]$

$$
\mathrm{Sh} \approx 1+0.26 \mathrm{Pe}^{1 / 2} \text {. }
$$

Before presenting the results of simulations of Eq. (21), we discuss the relevant scales for well-resolving the competition between shear and diffusion. Shear and diffusion balance at a scale $\ell_{\gamma} \sim \sqrt{D / \gamma}$, diverging for $\gamma \rightarrow 0$. Stationarity (in the infinite volume) is reached when the diffusive front becomes comparable with the scale $\ell_{\gamma}$, i.e., for times $\tau_{\gamma} \sim \ell_{\gamma}^{2} / D=\gamma^{-1}$, also diverging for $\gamma \rightarrow 0$. Thus $\ell_{\gamma}$ should be much smaller than the simulation box $L$, otherwise the effect of shear starts to be effective over time scales for which the absorber is also interacting with its periodic images. The requirement $\ell_{\gamma} \ll L$ implies a constraint on the smallest shear rate that can be used, i.e., $\gamma \gg D / L^{2}$. Moreover, since we are interested in testing the prediction (22) for $\mathrm{Pe}=\gamma R^{2} / D \ll 1$, we end up with the requirements $D / L^{2} \ll \gamma \ll D / R^{2}$, which can be reexpressed as $R^{2} / D=\tau_{d} \ll \tau_{\gamma} \ll \tau_{L}=L^{2} / D$ and $R \ll \ell_{\gamma} \ll L$ in the time and scale domain, respectively. The limitation on the smallest value of $\gamma$ is well evident from Fig. 12, where we

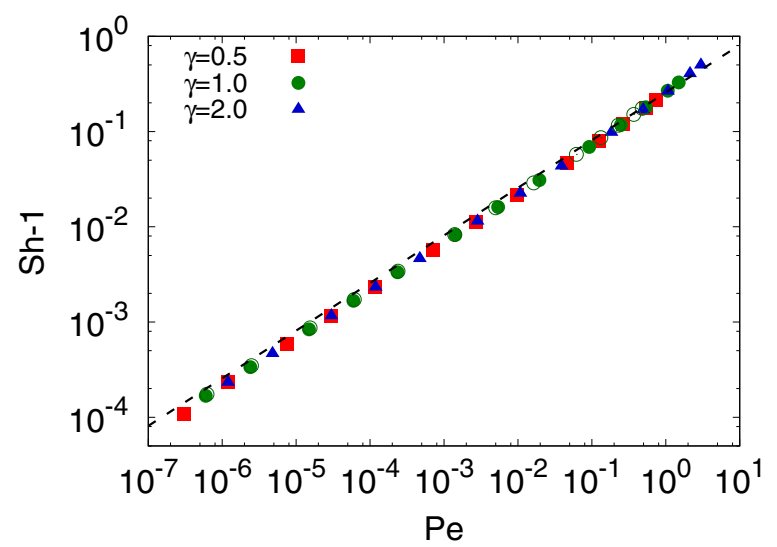

FIG. 13. Shear contribution to the Sherwood number Sh as a function of Peclet number Pe for numerical simulations at two different resolutions: $M=64$ (filled symbols) and $M=128$ (empty symbols). The line represents the theoretical behavior (22). The Peclet number Pe is changed by fixing the shear rate $\gamma$ and varying the radius $R$.

show the time evolution of the uptake rate, $\kappa(t)$, at varying $\gamma$ when $D$ and $R$ are fixed. For time $t \ll \tau_{\gamma}, \kappa(t)$ is essentially indistinguishable from that obtained in the diffusive case $(\gamma=$ $0)$. For $\gamma \lesssim 0.01, \tau_{\gamma}$ is comparable with the time at which the decay induced by the absorber periodic images becomes effective. Figure 12 also shows that, due to the shear, the time behavior of the uptake rate is quite different from the Smoluchowski (diffusive) result, Eq. (6). As a consequence, we cannot exploit (6) to fit the rate, as was previously done. Without a theoretical prediction for $\kappa(t)$, we can extract the (infinite volume) uptake rate constant using Eq. (5). Assuming a constant uptake rate $\kappa$, the mean concentration should decay linearly in time as $C(t)=C_{0}-(\kappa / V) t$ with $V=L^{3}=(2 \pi)^{3}$. The above functional form should be fitted in the time interval $\tau_{\gamma}<t<\tau_{L}$, when the disturbance induced by the shear is well-developed and (quasi)stationary. To test the prediction (22), we proceed as follows. Given the diffusion coefficient $D$, we fixed the shear rate $\gamma$ at three representative values, such that $\ell_{\gamma}$ is well resolved by the numerical grid and $\ell_{\gamma} \ll L$. Then we explored different values of the Peclet number Pe by varying the particle radius $R$ (viz., the absorption rate $\beta$ ), but enforcing the constraint $\ell_{\gamma} \gg R$ to have a small Pe. We performed two series of simulations using grid resolution $M=64,128$. For each series of simulations, we explored a sufficiently wide range of values of the Peclet number, fitting the uptake rate constant as described above. As shown in Fig. 13, the excess Sherwood number, $\mathrm{Sh}-1$, as a function of Pe compares very well with (22).

\section{CONCLUSIONS}

We presented a numerical method for computing the nutrient uptake rate by small spherical particles immersed in a concentration field, in the diffusion-limited regime. The method, inspired by the force-coupling method, represents each particle as an effective sink of concentration and can in principle be used in the presence of a generic underlying flow, motile particles, and source terms for the concentration 
field. Moreover, a more complex reaction scheme can be easily implemented to mimic partial or saturable absorption.

By comparing the numerical results with exact results obtained from a multipole expansion method based on reexpansion formulas for solid harmonics, we have shown that the method, implemented here on a pseudospectral solver, is able to correctly reproduce the diffusive interactions among competing absorbers arranged in geometrical configurations of increasing complexity. As was discussed, the main limitation of the method resides in the possibility to resolve diffusive interactions at small distances, but this can be cured by increasing the resolution. Another limitation pertains to the large distances, but this is only due to the periodicity of the simulation domain and thus it does not concern the method itself.

The main advantages of the method are the scalability with the number of absorbers and the possibility to include the presence of an arbitrary flow, for which we show a benchmark in the case of a linear shear. These properties make our numerical method ideal for the study of problems possibly involving complex, turbulent flows, such as the efficiency of nutrient uptake by micro-organisms in the ocean. In future investigations, we plan to implement the presented method to particles transported by turbulent flows, backreacting on it and possibly equipped with self-propulsion.

\section{ACKNOWLEDGMENTS}

We acknowledge the European COST Action MP1305 "Flowing Matter."

\section{APPENDIX A: SMOLUCHOWSKI FORMULA}

The problem of diffusion-limited reaction was first studied by Smoluchowski [10] and then applied to the heat flow into a sphere with a constant temperature [55]. In the ecology of phytoplankton, the model was first introduced by Osborn [56]. In the absence of a flow, the uptake by a single spherical cell is controlled by the diffusion equation

$$
\partial_{t} \rho=D \nabla^{2} \rho
$$

where $D$ is the diffusivity, and the boundary conditions (for a perfect absorber) are $\rho=0$ at $r=R$ and $\rho=\rho_{\infty}$ as $r \rightarrow \infty$.

Using the Laplace transform, Eq. (A1) gives the solution

$$
\rho(r, t)=\rho_{\infty}\left[1-\frac{R}{r} \operatorname{Erfc}\left(\frac{r-R}{2 \sqrt{D t}}\right)\right] .
$$

The flux per unit area is $J(r, t)=-D \partial_{r} \rho$. Integrated over the solid angle at $r=R$, this gives the rate of nutrient flux entering into the cell surface, i.e., $\kappa(t)=J(R, t) 4 \pi R^{2}$. Therefore, from (A2), the uptake rate at the sphere is

$$
\kappa(t)=4 \pi D R \rho_{\infty}\left(1+\frac{R}{\sqrt{\pi D t}}\right) .
$$

In the limit of long times $t \rightarrow \infty, \kappa(t)$ reduces to the Smoluchowski constant rate $\kappa_{s}$.

\section{APPENDIX B: THE MEAN-FIELD THEORY OF ABSORPTION BY A SPHERICAL POTENTIAL}

Here we aim at solving the following equation:

$$
\nabla^{2} \varphi-k \Theta(b-|\boldsymbol{r}|) \varphi=0,
$$

with the boundary condition $\varphi(|\boldsymbol{r}| \rightarrow \infty)=\varphi_{\infty}$, and where $\Theta$ is the Heaviside function. The solution we are interested in is spherically symmetric, so, denoting $r=|\boldsymbol{r}|$, the equation we actually need to solve is

$$
\varphi^{\prime \prime}+2 \varphi^{\prime} / r-\varphi / \xi^{2}=0
$$

where $\xi=\sqrt{D / k}$ has dimensions of a length, and the prime denotes the derivative with respect to $r$. The general solution is [57]

$$
\varphi= \begin{cases}\frac{C_{1} \xi \sinh (r / \xi)+C_{2} \xi \cosh (r / \xi)}{r}, & r \leqslant b, \\ \frac{C_{3}}{r}+\varphi_{\infty}, & r \geqslant b .\end{cases}
$$

To avoid a singular solution in $r=0$, we impose $C_{2}=0$, while $C_{1}$ and $C_{3}$ can be fixed imposing continuity of $\varphi$ and its derivative in $r=b$. The final result is

$$
\varphi(r)= \begin{cases}\frac{\varphi_{\infty} \xi}{\cosh (b / \xi)} \frac{\sinh (r / \xi)}{r}, & r \leqslant b, \\ \varphi_{\infty}\left(1-\frac{b-\xi \tanh (b / \xi)}{r}\right), & r \geqslant b .\end{cases}
$$

For the results presented in the main text, we need to compute the flux on the surface of the sphere of radius $b$, which is simply obtained as

$$
\kappa=\left.4 \pi D r^{2} \frac{\partial \varphi}{\partial r}\right|_{r=b}=4 \pi D \varphi_{\infty}[b-\xi \tanh (b / \xi)] .
$$

\section{APPENDIX C: THE ABSORPTION BY A SPHERICAL SHELL POTENTIAL}

Here we aim at solving Eq. (19). Similarly to the case discussed in Appendix B [see Eq. (B3)], we have three regions with different solutions. In the interior of the shell, for $r<R_{s}-2 R$, we have the solution $\psi_{\mathrm{I}}=a_{1}+b_{1} \xi / r$, clearly $b_{1}=0$ due to the divergence at $r=0$. In the region $R_{s}-2 R<$ $r<R_{s}$, the solution is $\psi_{\mathrm{II}}=(\xi / r)\left(a_{2} e^{r / \xi}+b_{2} e^{-r / \xi}\right)$. In the outer region, $r>R_{s}$, the solution is $\psi_{\mathrm{III}}=a_{3}+b_{3} \xi / r$.

The boundary condition at infinity implies that $a_{3}=\psi_{\infty}$. Imposing the continuity of the solution and its derivative at $r=R_{s}-2 R$ and $r=R_{s}$, we obtain the remaining unknown constants. The final solution is

$$
\frac{\psi(x)}{\psi_{\infty}}= \begin{cases}\frac{1}{X_{1} \sinh (X)+\cosh (X)} & \text { (I) } \\ \frac{1}{x} \frac{X_{1} \cosh \left(x-X_{1}\right)+\sinh \left(x-X_{1}\right)}{X_{1} \sinh (X)+\cosh (X)} & \text { (II) } \\ 1-\frac{1}{x} \frac{\left(X_{1} X_{2}-1\right) \tanh (X)+X}{X_{1} \tanh (X)+1} & \text { (III) }\end{cases}
$$

where $x=r / \xi, X=2 R / \xi, X_{1}=\left(R_{s}-2 R\right) / \xi, X_{2}=R_{s} / \xi$, and the three regions correspond to $\mathrm{I}=\left[0, X_{1}\right], \mathrm{II}=\left[X_{1}, X_{2}\right]$, and III $=\left[X_{2}, \infty\right)$.

As before, the total uptake rate at $r=R_{s}$ is given by

$$
\kappa_{\mathrm{tot}}=\left.4 \pi D r^{2} \frac{\partial \psi}{\partial r}\right|_{r=R_{s}}=4 \pi D b_{3} \xi,
$$


where $b_{3}$ can be read from the term proportional to $1 / x$ in (C1) III.

\section{APPENDIX D: EXACT SOLUTION OF THE LAPLACE BOUNDARY PROBLEM WITH MANY DISCONNECTED SPHERICAL BOUNDARIES THROUGH ADDITION THEOREMS FOR SOLID HARMONICS}

In this appendix, we sketch the mathematical reasoning behind the exact solution of the Laplace equation with many disconnected sinks. For more details, see Ref. [15].

Let us pose the general problem of computing the stationary flux of a ligand-nutrient field toward a stationary distribution of $N$ spherical sinks, i.e., perfectly absorbing spheres $\Omega_{\alpha}$ of radius $R_{\alpha}(\alpha=1,2, \ldots, N)$. Let $\rho_{B}$ denote the (constant) bulk density of the nutrient field far from the sinks. The stationary normalized density $u(\mathbf{r})=\rho(\mathbf{r}) / \rho_{B}$ is the solution of the following boundary problem:

$$
\begin{aligned}
\nabla^{2} u & =0, \\
\left.u\right|_{\partial \Omega_{\alpha}} & =0 \text { for } \alpha=1,2, \ldots, N, \\
\lim _{r \rightarrow \infty} u & =1 .
\end{aligned}
$$

Once the Laplace equation is solved (see below for details), the intake rate can be computed as the total flux into the sink system, that is,

$$
k=\left.D \sum_{\alpha=1}^{N} \int_{\partial \Omega_{\alpha}} \frac{\partial u}{\partial r_{\alpha}}\right|_{\partial \Omega_{\alpha}} d S .
$$

To solve the Laplace equation in the assigned manifold, we introduce as many sets of spherical coordinate systems as there are spherical boundaries. The solution can then be written formally as an expansion in a series of irregular solid harmonics, namely

$$
\begin{aligned}
u & =1+\sum_{\alpha=1}^{N} u_{\alpha}=1+\sum_{\alpha=1}^{N} \sum_{n=0}^{\infty} \sum_{m=-n}^{n} B_{m n}^{\alpha} u_{m n}^{-}\left(\boldsymbol{r}_{\alpha}\right) \\
& =1+\sum_{\alpha=1}^{N} \sum_{n=0}^{\infty} \sum_{m=-n}^{n} B_{m n}^{\alpha}\left(\frac{r_{\alpha}}{R_{\alpha}}\right)^{-n-1} P_{n}^{m}\left(\cos \theta_{\alpha}\right) e^{i m \phi_{\alpha}},
\end{aligned}
$$

where $P_{n}^{m}$ are associated Legendre functions and $r_{\alpha}$ is the radial coordinate associated with the spherical system, which is centered on the $\alpha$ th sphere. The coefficients $B_{m n}^{\alpha}$ should be determined by imposing the boundary conditions (D1b). To do so, we can use known addition theorems for the spherical harmonics [38] to express the solution (D3) in all the $N$ different reference frames centered at each sphere. As a result, we obtain an infinite-dimensional system of linear equations for the unknown coefficients $B_{m n}^{\alpha}$,

$$
B_{g q}^{\alpha}+\left(\delta_{(g, q),(0,0)}+\sum_{n=0}^{\infty} \sum_{m=-n}^{n} \sum_{\beta=1, \beta \neq \alpha}^{N} B_{m n}^{\beta} W_{m, n}^{(\alpha, \beta, g, q)}\right)=0
$$

for all $\alpha=1,2, \ldots, N, q=1,2, \ldots \infty$, and $g=-q, \ldots, q$, with

$$
\begin{aligned}
W_{m, n}^{(\alpha, \beta, g, q)}= & (-1)^{q+g} \frac{(n-m+q+g) !}{(n-m) !(q+g) !} \\
& \times\left(\frac{R_{\alpha}^{q} R_{\beta}^{n+1}}{L_{\beta \alpha}^{n+q+1}}\right) Z_{m-g, n+q}\left(\boldsymbol{L}_{\beta \alpha}\right),
\end{aligned}
$$

where $\boldsymbol{L}_{\beta \alpha}$ is the vector that connects the centers of $\Omega_{\beta}$ and $\Omega_{\alpha}$ in the direction $\Omega_{\beta} \rightarrow \Omega_{\alpha}$, and $Z_{m \ell}$ is defined as

$$
Z_{m \ell}(\theta, \phi)=P_{\ell}^{m}(\cos \theta) e^{i m \phi} .
$$

To solve the above system, one needs to truncate the sum on $n$ in Eq. (D4) by including a finite number of multipoles, $N_{t}$, which should be chosen so as to attain the desired accuracy. Recalling the definition (D2), the intake rate $k$ can be computed explicitly. This gives

$$
k \equiv \sum_{\alpha=1}^{N} k_{\alpha}=-\sum_{\alpha=1}^{N} k_{S_{\alpha}} B_{00}^{\alpha},
$$

where $k_{S_{\alpha}}=4 \pi D R_{\alpha}$ is the Smoluchowski rate constant for an isolated sphere of encounter radius $R_{\alpha}$ (= radius of the absorber + radius of the ligand-nutrient). In the simplified case of equal sinks, $R_{\alpha}=a \forall \alpha$, this expression simplifies to

$$
\frac{k}{N k_{S}}=-\frac{1}{N} \sum_{\alpha=1}^{N} B_{00}^{\alpha}
$$

[1] S. A. Rice, Diffusion-Limited Reactions (Elsevier, Amsterdam, 1985), Vol. 25.

[2] H. C. Berg, Random Walks in Biology (Princeton University Press, Princeton, NJ, 1993).

[3] L. Karp-Boss, E. Boss, P. Jumars, et al., Oceanogr. Mar. Biol. 34, 71-108 (1996).

[4] T. Kiørboe, A Mechanistic Approach to Plankton Ecology (Princeton University Press, Princeton, NJ, 2008).

[5] L. G. Leal, Advanced Transport Phenomena: Fluid Mechanics and Convective Transport Processes (Cambridge University Press, Cambridge, 2007).

[6] Z. Neufeld and E. Hernández-García, Chemical and Biological Processes in Fluid Flows: A Dynamical Systems Approach (World Scientific, Singapore, 2009).
[7] S. Fournier-Bidoz, A. C. Arsenault, I. Manners, and G. A. Ozin, Chem. Commun. 4, 441 (2005).

[8] G. A. Ozin, I. Manners, S. Fournier-Bidoz, and A. Arsenault, Adv. Mater. 17, 3011 (2005).

[9] R. Golestanian, T. Liverpool, and A. Ajdari, New J. Phys. 9, 126 (2007).

[10] M. V. Smoluchowski, Kolloid-Zeitschrift 21, 98 (1917).

[11] S. Traytak, Chem. Phys. Lett. 197, 247 (1992).

[12] S. Traytak, Chem. Phys. 193, 351 (1995).

[13] N. Dorsaz, C. De Michele, F. Piazza, P. De Los Rios, and G. Foffi, Phys. Rev. Lett. 105, 120601 (2010).

[14] M. O. Lavrentovich, J. H. Koschwanez, and D. R. Nelson, Phys. Rev. E 87, 062703 (2013). 
[15] M. Galanti, D. Fanelli, S. D. Traytak, and F. Piazza, Phys. Chem. Chem. Phys. 18, 15950 (2016).

[16] M. Linkès, M. Martins Afonso, P. Fede, J. Morchain, and P. Schmitz, Chem. Eng. Sci. 81, 8 (2012).

[17] Z. Kalay, T. K. Fujiwara, and A. Kusumi, PLoS One 7, e32948 (2012).

[18] R. Grima and S. Schnell, Biophys. Chem. 124, 1 (2006).

[19] A. Kusumi, T. K. Fujiwara, R. Chadda, M. Xie, T. A. Tsunoyama, Z. Kalay, R. S. Kasai, and K. G. N. Suzuki, Annu. Rev. Cell Dev. Biol. 28, 215 (2012).

[20] V. Sourjik, Trends Microbiol. 12, 569 (2004).

[21] K. M. S. O'Connell, A. S. Rolig, J. D. Whitesell, and M. M. Tamkun, J. Neurosci. 26, 9609 (2006).

[22] A. Kusumi, T. K. Fujiwara, N. Morone, K. J. Yoshida, R. Chadda, M. Xie, R. S. Kasai, and K. G. N. Suzuki, Semin. Cell Dev. Biol. 23, 126 (2012).

[23] Š. Bálint, and M. L. Dustin, eLife 6, e25375 (2017).

[24] A. K. Shukla, G. Singh, and E. Ghosh, Trends Biochem. Sci. 39, 594 (2014).

[25] Z. Feng and E. Michaelides, Int. J. Heat Mass Transf. 52, 777 (2009).

[26] C. Eun, P. M. Kekenes-Huskey, and J. A. McCammon, J. Chem. Phys. 139, 044117 (2013).

[27] J. Schöneberg and F. Noé, PLoS One 8, e74261 (2013).

[28] J. Biedermann, A. Ullrich, J. Schöneberg, and F. Noé, Biophys. J. 108, 457 (2015).

[29] B. Felderhof, J. Deutch, and U. Titulaer, J. Chem. Phys. 76, 4178 (1982).

[30] M. Muthukumar, J. Chem. Phys. 76, 2667 (1982).

[31] R. Bonnecaze and J. F. Brady, J. Chem. Phys. 94, 537 (1991).

[32] P. M. Richards, Phys. Rev. B 35, 248 (1987).

[33] S. B. Lee, I. C. Kim, C. A. Miller, and S. Torquato, Phys. Rev. B 39, 11833 (1989).

[34] H.-K. Tsao, S.-Y. Lu, and C.-Y. Tseng, J. Chem. Phys. 115, 3827 (2001).

[35] A. Donev, V. Bulatov, T. Oppelstrup, G. Gilmer, B. Sadigh, and M. Kalos, J. Comput. Phys. 229, 3214 (2010).

[36] D. Grebenkov, First-Passage Phenomena and Their Applications (World Scientific, Singapore, 2014).
[37] S. D. Traytak, Diffusion Fundamentals: Leizig 1, 176 (2005).

[38] P. M. Morse and H. Feshbach, Methods of Theoretical Physics (Technology, Cambdrige, MA, 1946).

[39] E. Gordeliy, S. L. Crouch, and S. G. Mogilevskaya, Int. J. Numer. Methods Eng. 77, 751 (2009).

[40] G. Oshanin, O. Bénichou, M. Coppey, and M. Moreau, Phys. Rev. E 66, 060101(R) (2002).

[41] S. B. Yuste, G. Oshanin, K. Lindenberg, O. Bénichou, and J. Klafter, Phys. Rev. E 78, 021105 (2008).

[42] R. A. Lambert, F. Picano, W.-P. Breugem, and L. Brandt, J. Fluid Mech. 733, 528 (2013).

[43] M. M. Musielak, L. Karp-Boss, P. A. Jumars, and L. J. Fauci, J. Fluid Mech. 638, 401 (2009).

[44] C. S. Peskin, Acta Numer. 11, 479 (2002).

[45] M. Maxey, B. Patel, E. Chang, and L.-P. Wang, Fluid Dyn. Res. 20, 143 (1997).

[46] S. Lomholt and M. R. Maxey, J. Comput. Phys. 184, 381 (2003).

[47] S. Lomholt, B. Stenum, and M. Maxey, Int. J. Multiphase Flow 28, 225 (2002).

[48] A. Pal Singh Bhalla, B. E. Griffith, N. A. Patankar, and A. Donev, J. Chem. Phys. 139, 214112 (2013).

[49] N. A. Frankel and A. Acrivos, Phys. Fluids 11, 1913 (1968).

[50] R. Samson and J. Deutch, J. Chem. Phys. 67, 847 (1999).

[51] F. Piazza, P. De Los Rios, D. Fanelli, L. Bongini, and U. Skoglund, Eur. Biophys. J. 34, 899 (2005).

[52] S. D. Traytak, Phys. Biol. 10, 045009 (2013).

[53] S. M. Miller, Nature Education 3, 65 (2010).

[54] N. A. Frankel and A. Acrivos (Ref. [49]) attained the result $\mathrm{Nu}=2+c^{\prime} \mathrm{Pe}^{\prime 1 / 2}$ by defining $\mathrm{Nu}$ (the homolog of Sh for the heat transfer) based on the particle diameter, $\mathrm{Pe}^{\prime}=2 R^{2} \gamma / D$, and with $c^{\prime}=0.9104 / \sqrt{2 \pi} \simeq 0.36$. Transforming in our variables $\mathrm{Nu}=2 \mathrm{Sh}$ and $\mathrm{Pe}^{\prime}=2 \mathrm{Pe}$, we obtain the relation $\mathrm{Sh}=1+$ $c \mathrm{Pe}^{1 / 2}$ with $c=c^{\prime} / \sqrt{2} \simeq 0.26$.

[55] H. S. Carslaw and J. C. Jaeger (Clarendon, Oxford, 1959).

[56] T. Osborn, J. Plankton Res. 18, 185 (1996).

[57] V. Zaitsev and A. Polyanin, Handbook of Exact Solutions for Ordinary Differential Equations (Chapman \& Hall/CRC, London, 2002). 